Mercedes Díaz-Somoano*, Adriana Popa, Marta Rumayor, M. Antonia López-Antón, M. Rosa Martínez-Tarazona and Gheorghe Ilia

\title{
Gaseous mercury behaviour in the presence of functionalized styrene-divinylbenzene copolymers
}

\begin{abstract}
Two styrene- $6.7 \%$ divinylbenzene copolymers functionalized with aminophosphonate groups and phosphonic acid groups by means of "one-pot" reactions were evaluated for gaseous mercury removal. The results were compared with those obtained using a commercial activated carbon. These materials exhibit a significant capacity for mercury oxidation (13-25\%) with low mercury capture (9-30 $\left.\mu \mathrm{g} \mathrm{g}^{-1}\right)$. The mercury retention capacity was observed to decrease when acid gases are present in the gas atmosphere. The highest retention capacity corresponded to the highest oxidation ratio and was obtained using the AMINOPHOS sample. These results suggest a mercury oxidation and a subsequent chemical adsorption mechanism in which the amino groups play a role in mercury capture.
\end{abstract}

Keywords: functionalized S-6.7\%DVB copolymers; mercury adsorption; mercury oxidation; POC-2014.

DOI 10.1515/pac-2014-0614

\section{Introduction}

Mercury is a highly toxic pollutant of global concern [1]. Exposure to mercury can affect the human nervous system and harm the brain, heart, kidneys, lungs, and immune system. Gaseous elemental mercury (GEM) is emitted into the atmosphere from both natural and anthropogenic sources, and has an atmospheric residence time of $0.5-2$ years, allowing it to be transported over great distances [2, 3]. In January 2013 governments took a major step forward to counteracting the health and environmental impact of mercury by signing a global Convention agreement covering emissions and releases [4]. Coal combustion has been identified as one of the major sources of anthropogenic mercury in the environment. In Europe, according to the latest available data 28.1 tons of mercury were emitted to the air by different industrial activities in 2012, with a share of 55.4\% (15.6 tons) coming from thermal power stations and other combustion installations. [5]. The reduction of mercury emissions from these processes is becoming mandatory.

Installed air pollution control technologies such as particle control devices (ESP, baghouses...) and wet flue gas desulphurization systems can be effective for mercury removal. A wide range of removal efficiencies

Article note: A collection of invited papers based on presentations at the 15th International Conference on Polymers and Organic Chemistry (POC-2014), Timisoara, Romania, 10-13 June 2014.

*Corresponding author: Mercedes Díaz-Somoano, Instituto Nacional del Carbón-CSIC, Francisco Pintado Fe 26, 33011 Oviedo, Spain, e-mail: mercedes@incar.csic.es

Adriana Popa and Gheorghe Ilia: Institute of Chemistry Timisoara of Romanian Academy, 24 Mihai Viteazu Av., 300223

Timisoara, Romania

Marta Rumayor, M. Antonia López-Antón and M. Rosa Martínez-Tarazona: Instituto Nacional del Carbón-CSIC, Francisco Pintado Fe 26, 33011 Oviedo, Spain

(C) 2014 IUPAC \& De Gruyter 
has been reported depending on the type of coal, combustion parameters and installed control devices. The most widely used technology for mercury control is activated carbon (AC) injection into the gas stream. The main handicap of this technology is the high cost of the process. For this reason, other solids are being evaluated for gaseous mercury capture. Due to the volatility of mercury, original activated carbon is not very efficient in capturing elemental mercury especially at the typical temperature of flue gas cleaning. Mercury adsorption on activated carbon can be enhanced by the introduction of active sites (sulfur, chlorine, iodine...) [6-8] onto the solid surface. In addition, the injection of activated carbons that are later retained in the particle control devices compromises the quality of the fly ashes for future applications. In addition, most of the above-mentioned systems are limited by the oxidation state of mercury and mainly performed for oxidized mercury species and not for elemental mercury. For these reasons new solids are being investigated.

Chelating polymers have been widely used for metal ions removal during wastewater treatments. They offer a great adsorption capacity and selectivity and they can be regenerated [9]. However no sound knowledge of the gas-solid interactions with these materials is available. Previous studies carried out at INCAR-CSIC have proven that a chelating resin can also be effective for the selective adsorption of gaseous mercury species [10].

In this work two functionalized styrene-divinylbenzene copolymer samples: aminophosphonate grafted onto a styrene-divinylbenzene copolymer (AMINOPHOS) and hydroxyphosphonic acids grafted onto a styrene-divinylbenzene copolymer (PHOS) were synthesized by means of a "one-pot" reaction [11, 12]. This type of synthesis has been described in previous studies [11-15]. The functionalized resins were characterized by FT-IR spectroscopy, thermogravimetric analysis (TGA) and scanning electron microscopy (SEM). Gaseous mercury capture by the modified poly(styrene-co-divinylbenzene) was tested using a lab-scale device and the influence of gas composition on the mercury adsorption capacity was investigated. On the basis of the characterization of the post-retention solid an interaction mechanism will be proposed.

\section{Experimental}

\section{Materials}

A Chloromethylated styrene-divinylbenzene copolymer supplied by Purolite Romania (S-6.7\%DVB, \%Cl=14.22, $\mathrm{GF}=4.01 \mathrm{mmol} \mathrm{Cl} /$ gram. copolymer) was used as starting material together with a poly(styrene-co-divinybenzene) grafted with aldehyde groups synthesized in our laboratory [12, 16]. The percentage of divinyl benzene in the copolymers is the same for both cases.

\section{Synthesis of aminophosphonate grafted on copolymer (AMINOPHOS)}

The synthesis of the aminophosphonate functionalized on styrene-6.7\%divinylbenzene was performed according to a method described previously [11]. A mixture of $5 \mathrm{~g}$ of benzaldehyde grafted onto a styrenedivinylbenzene copolymer with $2.93 \mathrm{mmols}$-CHO /gram of copolymer and $50 \mathrm{~mL}$ THF (tetrahydrofuran) was kept under stirring for $2 \mathrm{~h}$ at room temperature to allow the copolymer beads to swell then phosphite and amine were added. The -CHO group: diethylphosphite: propylamine molar ratio was 1:1:1. The final mixture was maintained under stirring for $20 \mathrm{~h}$ at a temperature of $60^{\circ} \mathrm{C}$. After cooling, the polymer beads were separated by filtration, washed with ethanol $(3 \times 20 \mathrm{~mL}), 1,2$-dichloromethane $(3 \times 20 \mathrm{~mL})$ and ethyl ether $(3 \times 20$ $\mathrm{mL}$ ), and dried at $50{ }^{\circ} \mathrm{C}$ for $24 \mathrm{~h}$.

\section{One-pot synthesis of hydroxyphosphonic acid grafted on styrene-divinylbenzene copolymer (PHOS)}

The synthesis of the hydroxyphosphonic acid functionalized on styrene $-6.7 \%$ divinylbenzene was performed according to a method described previously [12]. A mixture of $5 \mathrm{~g}$ of aldehyde grafted onto styrene- 
divinylbenzene copolymer, phosphorous acid, $\mathrm{K}_{3} \mathrm{PO}_{4}$ as catalyst and $50 \mathrm{~mL}$ tetrahydrofuran was maintained under stirring for $35 \mathrm{~h}$ at $55^{\circ} \mathrm{C}$. The benzaldehyde group: phosphorous acid: $\mathrm{K}_{3} \mathrm{PO}_{4}$ molar ratio was 1:1.5:0.016. After cooling, the polymer beads were separated by filtration, washed with distilled water, methanol, acetone and diethyl ether and dried at $50{ }^{\circ} \mathrm{C}$ for $24 \mathrm{~h}$.

\section{Elemental mercury removal in gas phase}

The experimental lab scale device used for this purpose consisted of a glass reactor into which the solid was placed to form a fixed bed. The sorbent bed was prepared by mixing $20 \mathrm{mg}$ of copolymer with $60 \mathrm{mg}$ of sand. The temperature was kept at $150{ }^{\circ} \mathrm{C}$. Elemental mercury $\left(\mathrm{Hg}^{0}\right)$ in gas phase was obtained from a permeation tube. The concentration of $\mathrm{Hg}$ in gas phase was $100 \mu \mathrm{g} \mathrm{m}^{-3}$. A gas stream $(500 \mathrm{~mL} / \mathrm{min})$ was used to transport the mercury species through the sorbent. Two gaseous atmospheres were employed. A synthetic gas mixture containing compounds obtained from a coal oxy-combustion process $\left(4 \% \mathrm{O}_{2}, 0.1 \% \mathrm{NO}, 0.1 \% \mathrm{SO}_{2}, 12 \% \mathrm{H}_{2} \mathrm{O}\right.$, $100 \mathrm{ppm} \mathrm{NO}{ }_{2}, 25 \mathrm{ppm} \mathrm{HCl}, 20 \% \mathrm{~N}_{2}, 64 \% \mathrm{CO}_{2}$ ) was passed through the reactor. For interpretation and comparison purposes an air atmosphere was also tested. Oxy-fuel combustion is one of the emerging $\mathrm{CO}_{2}$ capture and storage technologies for coal-fired power generation. In oxy-fuel technology, coal is burned with a mixture of oxygen and recirculated flue gas, instead of air. The main components in the flue gas are $\mathrm{CO}_{2}, \mathrm{~N}_{2}, \mathrm{H}_{2} \mathrm{O}$ and $\mathrm{O}_{2}$.

Breakdown curves were recorded using a continuous mercury monitor (VM-3000). The possible oxidation of gaseous mercury was evaluated by capturing the oxidized mercury in an adsorption resin located prior to the continuous mercury analyzer. For this purpose a Dowex ${ }^{\circledR} 1 \times 8$ chloride form resin was placed at the exit of the reactor. Dowex is an ion exchanger designed specifically for the selective extraction of mercury (II) species. It was analyzed at the end of the retention experiments by means of an automatic mercury analyser (AMA 254) to determine the amount of mercury that had been oxidized.

After the mercury removal experiments, mercury speciation by thermal desorption (TD) of the solid samples was carried out. A continuous mercury analyzer (RA-915) coupled to a furnace (PYRO-915), both from Lumex, were used for the TD procedure [17]. The temperature of the equipment was continuously monitored by means of a thermocouple. The parameters used for the selective desorption were previously optimised. The mercury compounds desorbed from the sample were conducted through the chambers in a stream of air, at a flow rate of $11 \mathrm{~min}^{-1}$, the heating rate from room temperature to $750{ }^{\circ} \mathrm{C}$ was $50{ }^{\circ} \mathrm{C} \mathrm{min}{ }^{-1}$. About $100 \mathrm{mg}$ of sample was used for each thermo desorption analysis. The mercury species were identified according to temperature they were released from the sample and by comparison with the desorption temperatures characteristic of several reference mercury compounds collected from the literature [17].

\section{Results and discussion}

\section{Synthesis of AMINOPHOS and PHOS and characterization}

A chloromethylated styrene-divinylbenzene copolymer $(14.22 \% \mathrm{Cl})$ was used as the starting material in all the syntheses. An intermediate styrene-divinylbenzene coplymers grafted with aldehyde groups was synthesized in our laboratory $[12,16]$.

The copolymers functionalized with aminophosphonate groups were synthesized according to Scheme 1:

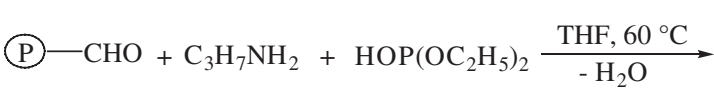<smiles>CCCNC([PH2+])CCC</smiles>

Scheme 1 The synthesis of the AMINOPHOS sample. 
The copolymers functionalized with phosphonic acid groups were synthesized according to Scheme 2:

The fraction of repeat units functionalized with aminophosphonate and $\alpha$-hydroxyphosphonic acid groups was determined by using the statistical structure of the repetitive unit of functionalized copolymers $[1,2]$ presented in Scheme $3(a, b)$.

The main characteristics of the aminophosphonate groups and $\alpha$-hydroxyphosphonic acid groups grafted onto a polymeric support by means of "one-pot" polymer-analogous reactions are given in Table 1 . The notation used was: Ff, - aminophosphonate groups/ $\alpha$-hydroxyphosphonic acid groups; Fi, $-\mathrm{CHO}$ groups; $\mathrm{r}$, fraction of divinylbenzen (DVB) units; $\mathrm{x}-\mathrm{y}$, fraction of styrene units bearing pendant $-\mathrm{CH}_{2} \mathrm{Cl}$ remaining groups; $z$, fraction of styrene units bearing pendant - aminophosphonate groups $/ \alpha$-hydroxyphosphonic acid groups (Ff); \% - phosphorus percentage in the final copolymer; $\mathrm{GF}$ - functionalization degree [11, 12, 18]. The phosphorus content was determined according to the modified Schoninger method [18]. The phosphorus content of the functionalized copolymers was used in order to determine the fraction of styrene units bearing aminophosphonate/ $\alpha$-hydroxyphosphonic acid groups (Ff) and it was calculated using previously published equations $[11,12,18]$.

Confirmation of the formation of aminophosphonate groups and $\alpha$-hydroxyphosphonic acid groups on the styrene-6.7\%divinylbenzene copolymer is obtained in the FTIR spectra (Fig. 1.) On the basis of: (i) an absorption band at around $1220 \mathrm{~cm}^{-1}$ associated with the vibration of the $\mathrm{P}=\mathrm{O}$ bond, (ii) a characteristic $\mathrm{P}-\mathrm{OH}$

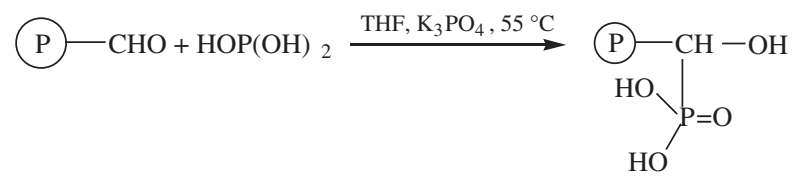

Scheme 2 The synthesis of the PHOS sample.<smiles>CCCCCC(CCC(Cc1ccc(CCl)cc1)CC(CC(C)C)c1ccc(C=O)cc1)c1ccccc1</smiles>

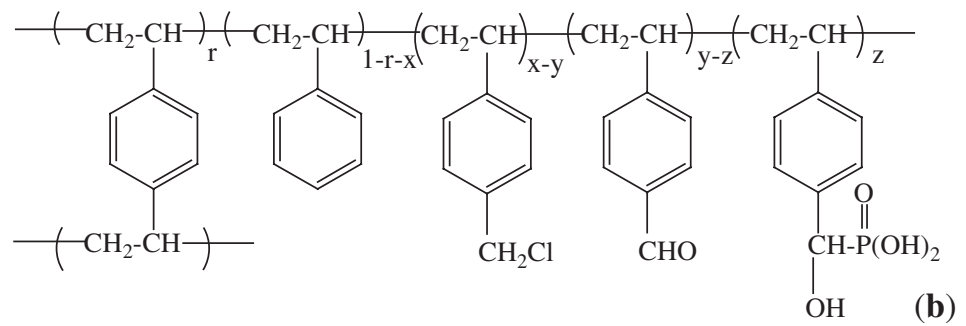

Scheme 3 Statistical structures of the repeat unit of the functionalized copolymers (a) aminophosphonate groups, (b) $\alpha$-hydroxyphosphonic acid groups.

Table 1 The main characteristics of the AMINOPHOS and PHOS samples.

\begin{tabular}{lrrrr}
\hline Sample & $\mathbf{P}($ wt, \%) & $\mathbf{x}-\mathbf{y}$ & $\mathbf{z}$ & $\mathbf{G}_{\mathrm{F}}{ }^{\mathrm{a}}$ (mmole/g) \\
\hline AMINOPHOS & 1.19 & 0.05 & 0.15 & 1.14 \\
PHOS & 1.15 & 0.05 & 0.14 & 1.13 \\
\hline
\end{tabular}

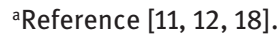




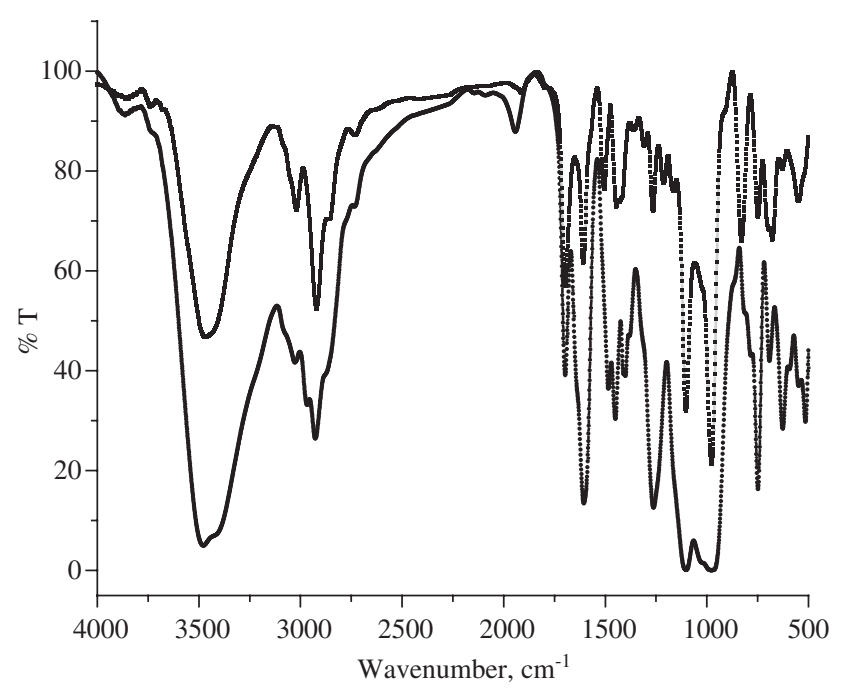

Fig. 1 FTIR spectra, where: phosphonic acid groups grafted onto S-6.7\%DVB (PHOS) -light gray; aminophosphonate groups grafted onto S-6.7\%DVB (AMINOPHOS) - black.

band appearing at around $1000 \mathrm{~cm}^{-1}$ and (iii) a medium intensity band at $1600 \mathrm{~cm}^{-1}$ assigned to the $\mathrm{P}(\mathrm{O}) \mathrm{OH}$ group.

The thermal behavior of the aminophosphonate and phosphonic acid resins (AMINOPHOS (a) and PHOS (b) samples) studied by thermogravimetric analysis in air, at a heating rate of $10^{\circ} \mathrm{C} / \mathrm{min}$ shows a characteristic "step weight" loss above $150^{\circ} \mathrm{C}$ (Fig. 2). These samples can therefore be used for the removal of elemental mercury in gas phase.

In the SEM image (Fig. 3) of the AMINOPHOS sample, macropores are clearly visible among the agglomerations of microspheres.

\section{Mercury adsorption and oxidation tests}

The mercury adsorption curves are shown in Fig. 4. The slope of the curve corresponding to PHOS is higher than the one obtained for the AMINOPHOS sample suggesting faster kinetic in this case. The Hg background level has not been reached for either solid indicating the possible oxidation of the mercury. The mercury retention capacities and oxidation rates are shown in Table 2. The retention capacities are lower than those obtained with the reference activated carbon (Filtracarb) [19] that is frequently used as sorbent specifically for mercury removal. However these values are comparable with the retention capacities of coal fly ashes [20] or biomass gasification chars [19] in an air atmosphere. This moderate retention capacity can be attributed to the absence of a carbonaceous matrix for mercury adsorption. However a significant degree of mercury oxidation was observed in both samples. The sample with higher retention capacity, AMINOPHOS, is the sample in which the maximum oxidation ratio was observed (Fig. 5). This suggests that oxidized mercury is most probable the captured species, as can was demonstrated subsequently in thermal desorption tests.

When the gaseous atmosphere contained reactive gases such as $\mathrm{SO}_{2}, \mathrm{NO}_{2}$ or $\mathrm{HCl}$, (Oxy-fuel combustion atmosphere), the mercury retention capacity and oxidized mercury ratio decreased. These results can be attributed to the competition reactions of the gaseous components for the active sites. This assumption is supported by the low mercury oxidation ratio found in this atmosphere. No mercury was oxidized and therefore no mercury was captured on the surface of the solid. In addition, during the mercury retention experiments it was observed that the solid copolymers turned into a light brown color. It was confirmed that this change was due to the interaction between the solid and the gaseous atmosphere.

Thermal desorption profiles obtained for the post-retention samples are shown in Fig. 6. Similar desorption profiles were obtained for the AMINOPHOS sample in both atmospheres (Fig. 6a and c). A major peak at 

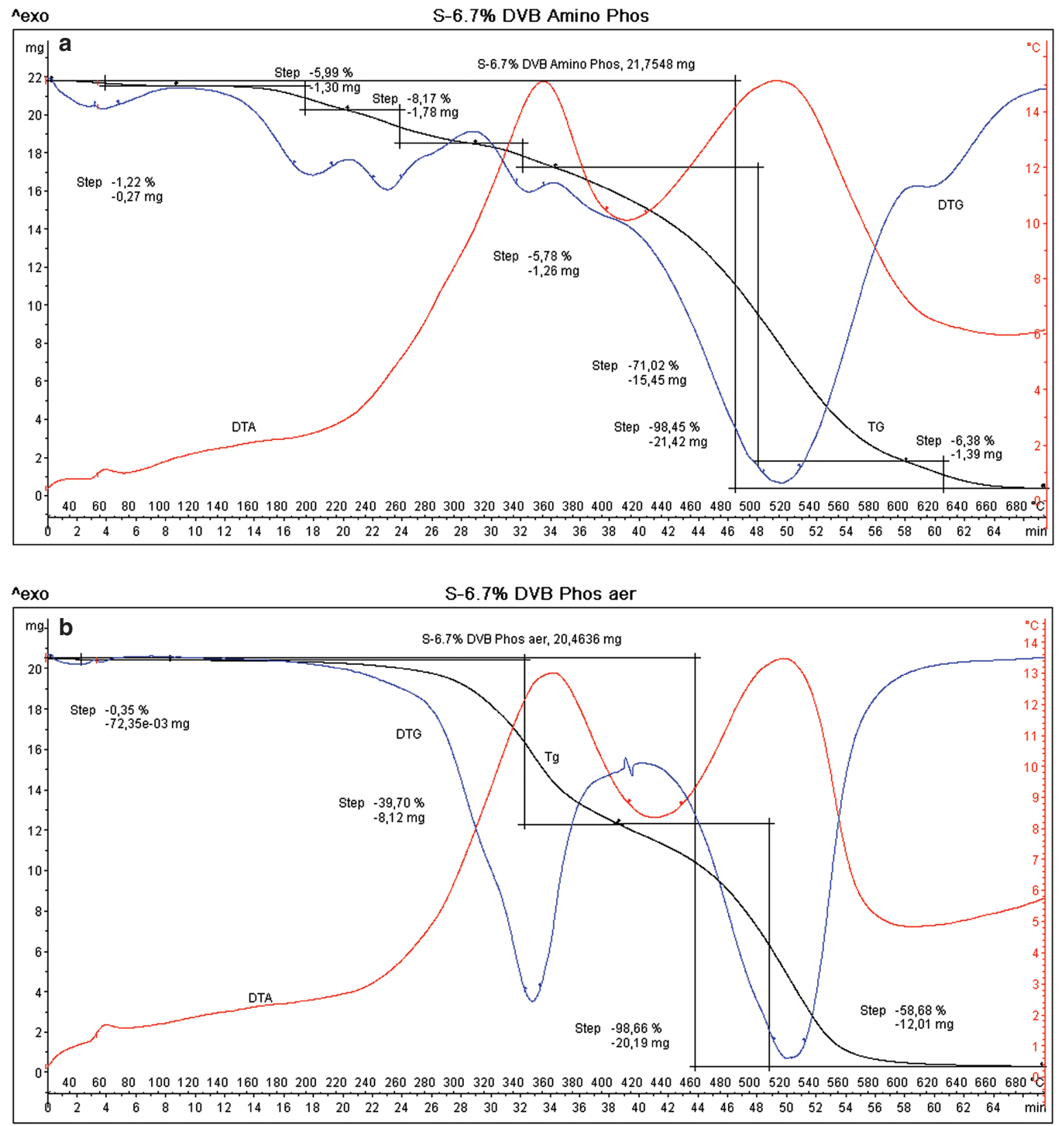

Fig. 2 Thermograms corresponding to the AMINOPHOS and PHOS samples.

$148{ }^{\circ} \mathrm{C}$ and a minor peak at $250^{\circ} \mathrm{C}$ are attributed to the presence of $\mathrm{HgCl}_{2}$ and $\mathrm{Hg}(\mathrm{CN})_{2}$ respectively. A simple peak with a maximum at $145^{\circ} \mathrm{C}$ was observed for the PHOS sample in the air atmosphere (Fig. 6b) corresponding to the formation of $\mathrm{HgCl}_{2}$. When this sample was tested under the oxyfuel combustion atmosphere two mercury species were identified by thermal desorption. Peaks at $160{ }^{\circ} \mathrm{C}$ and $450{ }^{\circ} \mathrm{C}$ corresponding to $\mathrm{HgCl}_{2}$ and $\mathrm{HgO}$, respectively, were observed (Fig. $6 \mathrm{~d}$ ). The formation of $\mathrm{HgCl}_{2}$ is due to the presence of residual chloromethyl pendant groups resulting from the sample preparation, see Table 1 (where: $x-y=0.05$, the fraction of styrene units bearing pendant $-\mathrm{CH}_{2} \mathrm{Cl}$ remaining groups). The formation of $\mathrm{Hg}(\mathrm{CN})_{2}$ and $\mathrm{HgO}$ confirms the capture of oxidized mercury.

All these results suggest the involvement of amino groups in the interaction of gaseous mercury with the functionalized copolymers. 


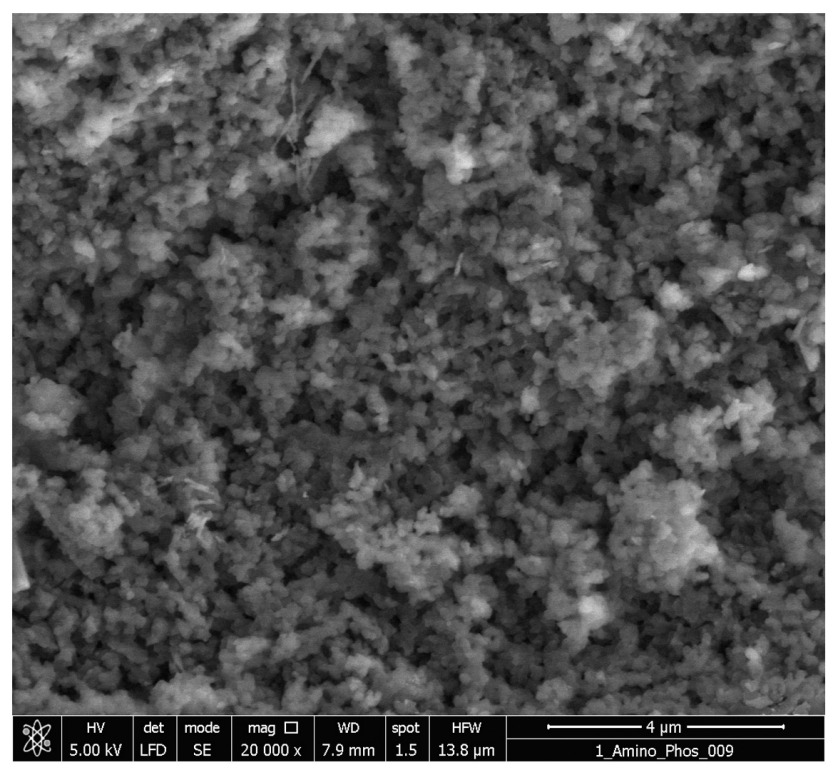

Fig. 3 SEM images of styrene-6.7\%divinylbenzene copolymer functionalized with aminophosphonate groups (code- AMINOPHOS).
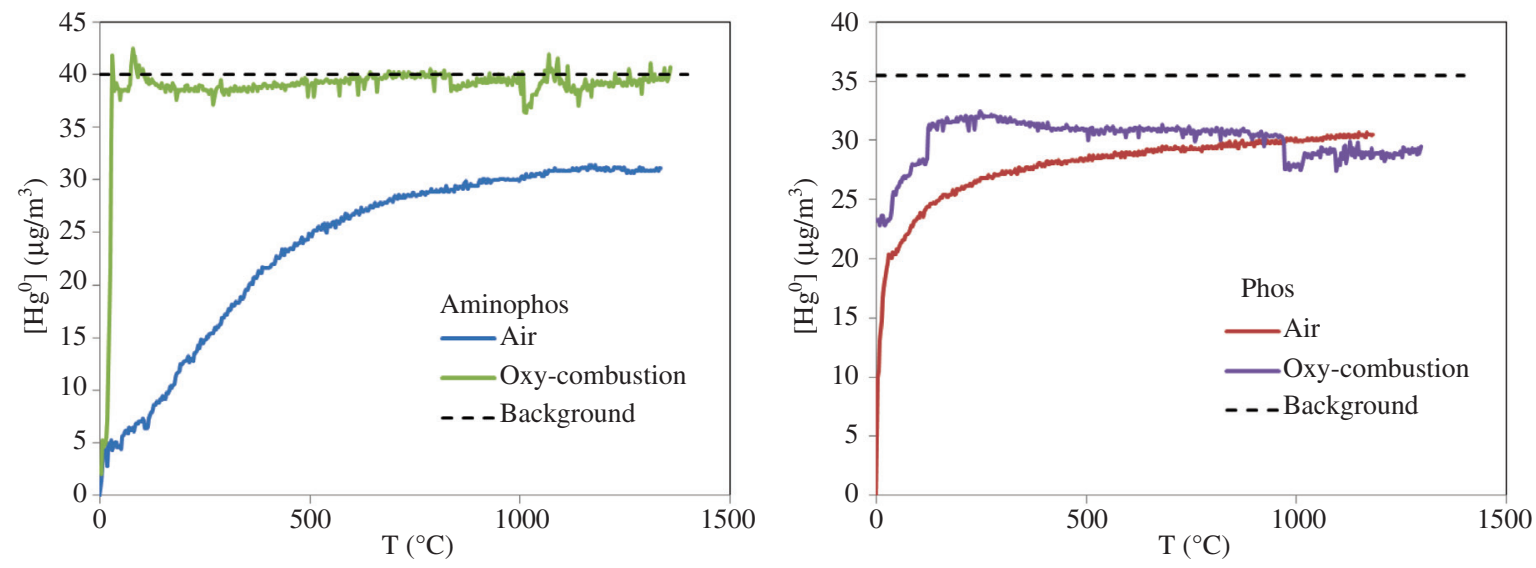

Fig. $4 \mathrm{Hg}$ adsorption using modified S-6.7\%DVB copolymers.

Table 2 Mercury retained and degree of mercury oxidation during experiments in an air atmosphere.

\begin{tabular}{lcccc}
\hline Sample & \multicolumn{2}{c}{ Hg Retention capacity $(\boldsymbol{\mu g} / \mathbf{g})$} & \multicolumn{2}{c}{ Hg oxidation (\%) } \\
\cline { 2 - 5 } & Air & Oxy-Comb. & Air & Oxy-Comb. \\
\cline { 2 - 5 } & 31 & $<1$ & 25 & 0 \\
AMINOPHOS & 8.9 & $<1$ & 13 & 10 \\
Coal Fly ash [20] & $1.1-20$ & n.a. & n.a. & n.a. \\
Biomass Gasification Char [19] & $1-570$ & n.a. & n.a. & n.a. \\
AC (Filtracarb) [19] & 844 & n.a. & n.a. & n.a. \\
\hline
\end{tabular}

Values for coal fly ash, biomass gasification char and activated carbon are shown as reference values.

\section{Conclusions}

In this study styrene-6.7\%divinylbenzene copolymers functionalized with propylamino-diethylphosphonate groups (S-6.7\%DVB AminoPhos) and alfa-hydroxyphosphonic acids grafted onto a styrene-divinylbenzene 

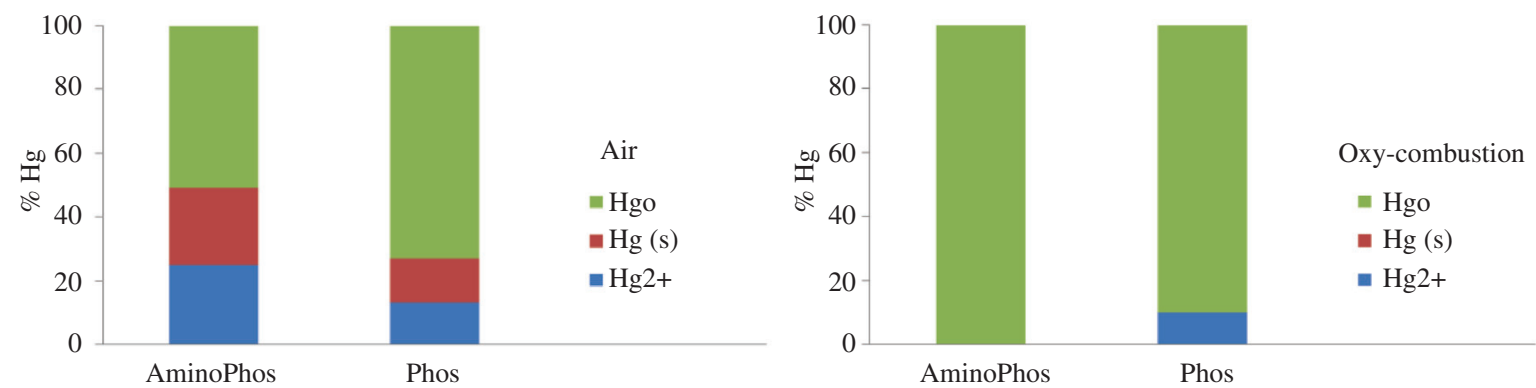

Fig. 5 Fate of $\mathrm{Hg}$ using S-6.7\%DVB copolymers as $\mathrm{Hg}$ adsorbents for gaseous $\mathrm{Hg}^{0}$.
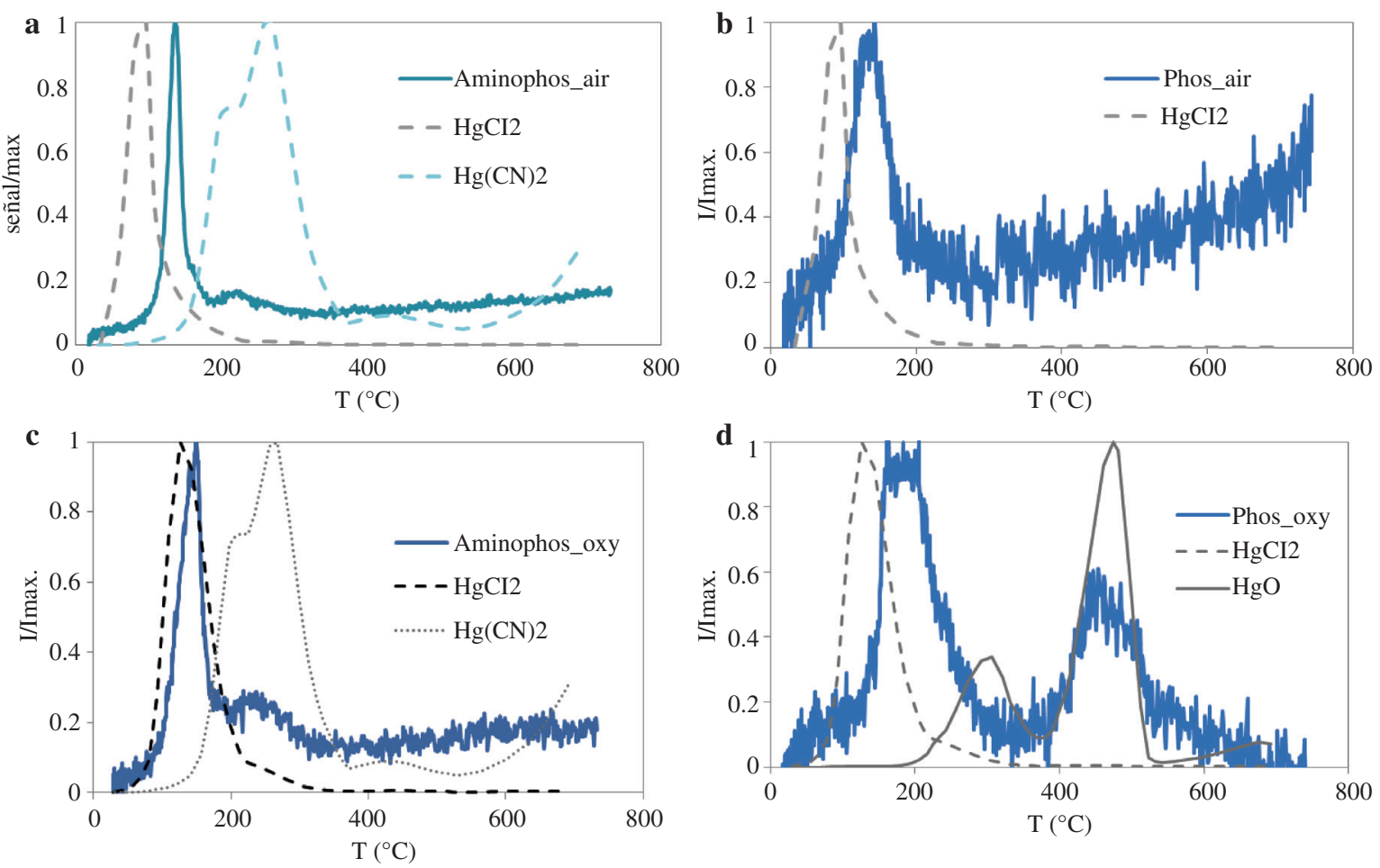

Fig. $6 \mathrm{Hg}$ TPD profiles for mercury speciation in S-6.7\%DVB copolymers. Continuous lines refer to tested samples in air and oxy-combustion atmospheres. Discontinuous lines refer to commercial mercury standards.

copolymer (S-6.7\%DVB Phos) were obtained with high degree of grafting around $80 \%$. These materials exhibit a significant capacity for mercury oxidation with low mercury capture. This fact is very interesting having into account that gaseous mercury control technologies are limited by the oxidation state of mercury being mostly performed for oxidized mercury species. The presence of amino groups in the solid enhances mercury oxidation and retention. The most probable interaction mechanism is chemical adsorption in which the amino groups are involved.

Acknowledgments: The authors are grateful for functionalized polymers that were obtained by partial financial support from Program no. 2, Project no. 2.4 from Romanian Academy, Institute of Chemistry Timisoara of Romanian Academy. Authors also thank the financial support for mercury adsorption studies provided by the Spanish National Council through the project CTM2011-22921. 


\section{References}

[1] G. Liu, Y. Cai, N. O’Driscoll. Wiley, New Jersey, ISBN: 978-0-470-57872-8, p. 574 (2012).

[2] C-J. Lin, S. O. Pehkonen. Atmos. Environ. 33, 2067 (1999).

[3] W. H. Schroeder, J. Munthe. Atmos. Environ. 32, 809 (1998).

[4] “Minamata Convention of Mercury"; January 2013; http://www.mercuryconvention.org.

[5] European Pollutant Release and Transfer Register (E-PRTR). http://prtr.ec.europa.eu.

[6] R. D. Vidic, D. P. Siler. Carbon 39, 3 (2001).

[7] K. Suresh Kumar Reddy, Ahmed Al Shoaibi, C. Srinivasakannan. Environ. Technol. 35, 18 (2014).

[8] E. C. Rupp, J. Wilcox. Fuel 117, 351 (2014).

[9] B. L. Rivas, I. M. Peric, C. Muñoz, R. Alvear. Polym. Bull. 68, 391 (2012).

[10] A. Fuente-Cuesta, M. Diaz-Somoano, M. A. Lopez-Anton, M. R. Martinez-Tarazona. Fuel 122, 218 (2014).

[11] A. Popa, V. Parvulescu, N. Plesu, S. Iliescu, C. M. Davidescu. Materiale Plastice 45, 10 (2008).

[12] A. Popa, S. G. Muntean, O. M. Paska, S. Iliescu, G. Ilia, Z. Zhang. Polym. Bull. 66, 419 (2011).

[13] A. Popa, C.-M. Davidescu, N. Petru, G. Ilia, A. Katsaros, K. D Demadis. Ind. Eng. Chem. Res. 47, 2010 (2008).

[14] C. M. Davidescu, M. Ciopec, A. Negrea, A. Popa, L. Lupa, E.-S. Dragan, R. Ardelean, G. Ilia, S. Iliescu. Polym. Bull. 70, 277 (2013).

[15] C. Pacurariu, G. Mihoc, A. Popa, S. G. Muntean, R. Ianos. Chem. Eng. J. 222, 218 (2013).

[16] A. Popa, G. Ilia, C.-M. Davidescu, S. Iliescu, N. Plesu, A. Pascariu, Z. Zhang. Polym. Bull. 57, 189 (2006).

[17] M. Rumayor M. Diaz-Somoano, M. A. Lopez-Anton, M. R. Martinez-Tarazona. Talanta 114, 318 (2013).

[18] A. Popa, C. M. Davidescu, R. Trif, Gh. Ilia, S. Iliescu, Gh. Dehelean. React. Funct. Polym. 55, 151 (2003).

[19] Final report MERCURYCAP project, http://bookshop.europa.eu/en/high-capacity-sorbents-and-optimisation-of-existingpollution-control-technology-for-mercury-capture-in-industrial-combustion-systems-mercurycap--pbKINA25318.

[20] P. Abad-Valle, M. A. Lopez-Anton, M. Diaz-Somoano, M. R. Martinez-Tarazona. Chem. Engin. J. 174, 86 (2011). 